\title{
Validation Study of a Novel Chest Tube Placement Device: Comparison of Procedure Time and Lung Injury Risk Between the JD Hook ${ }^{\circledR}$ Device and Conventional Chest Tube Placement Devices
}

\author{
Jameel Durrani
}

\begin{abstract}
Background: We tested the operational characteristics of JD Hook ${ }^{\circledR}$ prototype device and compared it to currently available direct chest tube insertion (non-Seldinger) devices in terms of procedure time, safety and risk of lung injury.
\end{abstract}

Methods: Two commercially chest tube devices including a 28 Fr chest tube with trocars, a $24 \mathrm{Fr}$ chest tube with a Kelly forceps clamp and JD Hook ${ }^{\circledR}$ prototype pleural access device were tested on standard thoracic manikin, for comparative procedure time and risk of lung injury.

Results: JD Hook ${ }^{\circledR}$ pleural access device showed the shortest procedure time with least risk of lung injury compared to conventional access devices. Both results were statistically significant.

Conclusions: JD Hook ${ }^{\circledR}$ device is a safer and faster access device compared to conventional chest tube access devices. Additional studies in animal and cadavers models are needed to validate these results for clinical applications.

Keywords: Chest tube; Pleural access; Pleural drainage catheter; Pleural effusion; Hemothorax; Pneumothorax; Chest wall trauma; Traumatic chest injuries

\section{Introduction}

Thoracic injuries account for $80 \%$ of preventable deaths in the battlefield [1]. In the civilian environment, chest injuries account for $20-25 \%$ of all trauma-related deaths in the USA [2]. Despite a clear life-saving advantage attributable to the procedure, current Combat Trauma Care (T-CCC) guidelines discour-

Manuscript submitted August 25, 2021, accepted September 26, 2021

Published online September 30, 2021

Department of Pulmonary and Critical Care Medicine, Doylestown Hospital, 595 W State Street, Doylestown. PA 18901, USA. Email: Jfdurrani@gmail.com

doi: https://doi.org/10.14740/jcs447 age field placement of chest tubes in the field [3] (primarily due to technical skills involved and concerns regarding wound size associated with conventional chest tube insertion methods), and encourage needle thoracostomies with 14G, 3.25" needles. Unfortunately, needle thoracostomy is a far inferior procedure compared to chest tubes, with a $58 \%$ failure rate even in controlled environments [4]. This data underscore the need for a simpler, faster and safer chest tube placement device that can be safely deployed in the field and trauma scenarios.

While the chest tubes have been used for hundreds of years, the techniques and devices available to access and drain the chest cavity have not significantly evolved over that time period. Current direct approaches (including spear-loaded, needle-loaded, or clamp-directed approach) are fraught with risks including lung injury, malposition, or entirely missing the access into the pleural space. Indirect approaches including guidewire or Seldinger techniques are not practical in the field environment due to the even higher number of steps (compared to direct insertion techniques) and additional technical skills involved in performing such interventions.

To review recent innovations in faster and safer access to chest cavity, we conducted a literature search on September 19, 2021, on PubMed with search terms including "Pleural catheter placement", "Chest tube placement devices", "Pleural cavity access device", "Chest tubes", and "Chest cavity access in the field". The search results were truncated for publication year 2010 to 2021. PubMed online database search yielded a total of 305 citations, which were individually reviewed for relevance to innovations in accessing chest cavity. Only one study by Fortune et al [5] met the criteria. This study described initial available data regarding a novel chest cavity access device with spring-loaded directional placement mechanism to optimize final placement of the chest tube within the chest cavity.

We hereby test the currently available conventional chest tube placement devices against a novel chest tube insertion device [6] (JD Hook ${ }^{\circledR}$ prototype device) that aims to enhance the speed, safety and precision of chest tube placement within the pleural space.

\section{Materials and Methods}

We tested the conventional chest tube devices (Argyll chest 


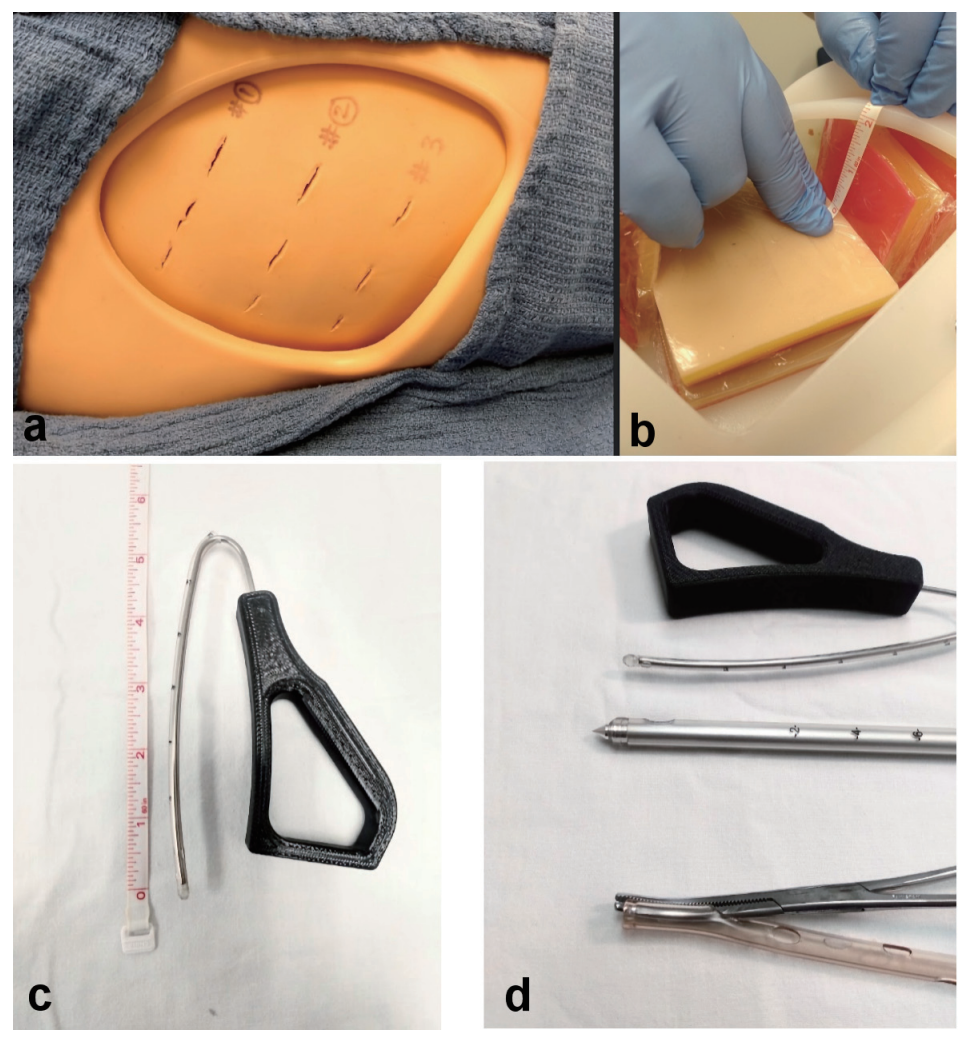

Figure 1. (a) Manikin setup for placement of chest tube devices. (b) Lung surface with visceral pleural surface cover placed $2 \mathrm{~cm}$ from the rib cage. (c) Insertable length of JD Hook ${ }^{\circledR}$ device housed within a 12 FR chest tube with blunt tip. (d) Close-up view of device tips used in the study.

tube, 28 Fr, with trocars, 24 FR Covidien chest tube with a Kelly forceps as introducer) and JD Hook ${ }^{\circledR}$ prototype device $[6,7]$ for procedure time and risk of lung surface injury. The experiment was conducted at the DeSales SIM lab [8], on standard thoracic manikin with removable ribcage flap modules and lung surface modules inserted on each side (Fig. 1).

Our aim was to compare the above mentioned devices on parameters including time required to insert the devices, and risk of injury to lung tissue.

Lung injury was defined by appearance of a puncture or visible disruption of a thin plastic film applied to a manikin lung tissue surface (to simulate visceral pleural/lung surface) positioned at 2-cm distance from the interior of rib cage (Fig. 1).

The procedure was performed by a single experienced operator. To minimize operator performance bias, the operator was blinded to intrathoracic views during the procedure, and continuous video (with digital timer display); recording was done while the study was in progress. The procedure was repeated three times for each device by the operator. Procedure time was calculated from the start of skin incision upto the final placement of chest tube as declared by the operator at end of procedure.

Preliminary steps that were common to all chest tube procedures were eliminated from the time calculation. These steps included surface preparation, administration of local analgesia, suturing the device in place, and attachment of drainage accessories.

Procedure was defined as completed, once the position of device was declared satisfactory and completed by the operator. The presence or absence of lung surface injury was visually recorded and scored by examining the inside of the manikin chest cavity at the completion of the experiment.

\section{Statistics}

We used one-way analysis of variance (ANOVA) for validity of repeat measurements. Data sets from the second and third trial were duplicated for the statistical application.

\section{Ethical compliance}

The study design was approved by the Institutional Review Committee of the JFD-PK Medical LLC. The study was conducted in compliance with the Helsinki Declaration. No human subjects or animals were used or harmed during the performance of this study.

\section{Results}

Device 1 (28 FR with trocar) yielded a mean insertion time of $40.4 \mathrm{~s}$, while device 2 (24 FR with Kelly forceps) yielded a mean insertion time of 59.6 s. JD Hook $^{\circledR}$ device yielded a 
Table 1. Chest Tube Insertion Times (in Seconds) for Each Device Over Three Consecutive Trials

\begin{tabular}{llll} 
& 28FR/trocar & 24FR/Kelly forceps & JD Hook $^{\circledR}$ \\
\hline Trial 1 & $46 \mathrm{~s}$ & $62 \mathrm{~s}$ & $23 \mathrm{~s}$ \\
Trial 2 & $39 \mathrm{~s}$ & $49 \mathrm{~s}$ & $25 \mathrm{~s}$ \\
Trial 3 & $39 \mathrm{~s}$ & $69 \mathrm{~s}$ & $29 \mathrm{~s}$ \\
Lung injury risk & $3 / 3(100 \%)$ & $1 / 3(33 \%)$ & $0 / 3(0 \%)$ \\
\hline
\end{tabular}

Bottom row represents lung injury risk score, showing number of times that the pleural surface contact was noted for each device out of three consecutive trials.

mean insertion time of $26.2 \mathrm{~s}$ (Table 1, Fig. 2). Application of one-way ANOVA test to the results yielded a $\mathrm{P}$ value of 0.000062 , consistent with a statistically significant difference in insertion times for different devices (Table 2).

Lung injury risk score was calculated by adding the number of pleural surface contacts noted for each device. Lung injury risk was highest for the trocar device at $100 \%$, followed by $33 \%$ for the device with Kelly forceps. Meanwhile JD Hook $^{\circledR}$ device showed no evidence of pleural surface contact with $0 \%$ score. Table 2 and Figure 3 summarize the lung injury risk score for each device.

\section{Discussion}

Traditional chest tubes/pleural access devices are plastic tubes of various sizes either loaded on to sharp edge needles/pointed spears, or pushed into the chest cavity with the help of curved serrated surgical clamps, (aka Kelly clamps/ forceps). All of these techniques have a forward facing tip towards the lung surface as they enter the pleural space, and need significant manual positioning and expertise to minimize damage to the lung and surrounding viscera. Such need for advanced manual skill sets and positioning expertise has limited the scope of chest cavity access devices to advanced practitioners and limited locations where the chest cavity can be accessed.

The JD Hook ${ }^{\circledR}$ device is an improved chest tube placement device with added safety features and ease of use in various environments by healthcare providers within a broad range of skill levels. Safety features are expected to reduce risk of lung injury and allow for quicker deployment of chest drainage catheters in field and trauma situations (Fig. 4). The JD Hook ${ }^{\circledR}$ device would allow for safe and more precise navigation and placement of the chest tube in a specific pleural cavity zone, based on intended use. The device has a curved metallic stylet $3 \mathrm{~mm}$ in diameter, with a blunt tip. The insertable length is 6 inches that is attached to an angulated grip segment. The introducer is loaded into the chest tube through a side hole and the blunt tip rests within the catheter tip. For optimal device performance, chest tubes with blunt radiopaque tips are recommended.

One of the goals of this study was to look into ways to extend the safety of chest tube placement beyond hospitals and emergencv rooms. into the field trauma scenes and combat sce-

28FR/Trocar $\square$ 24FR/Kelly forceps $\square$ JD Hook ${ }^{\circledR}$

80

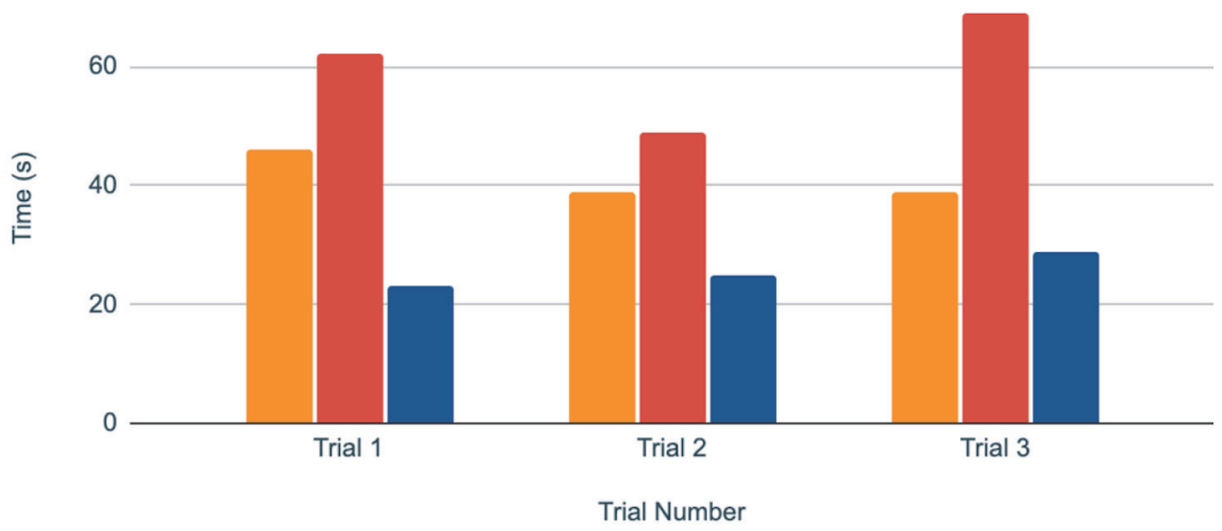

Figure 2. Chest tube insertion procedure times (in seconds) for each device over three consecutive trials.

Table 2. One-Way Repeated Measures ANOVA Test

\begin{tabular}{llll} 
& $\mathbf{2 8}$ FR/trocar & 24 FR/Kelly forceps & JD Hook $^{\circledR}$ device \\
\hline Mean procedure time & $40.4 \mathrm{~s}$ & $59.6 \mathrm{~s}$ & $26.2 \mathrm{~s}$ \\
Standard deviation & 3.13 & 10.08 & 2.68 \\
P value & 0.000062 & & \\
\hline
\end{tabular}

One-way repeated measures ANOVA test showed $\mathrm{P}$ value of 0.000062 . Trial 2 and trial 3 data were duplicated for statistical purposes. ANOVA: analysis of variance. 


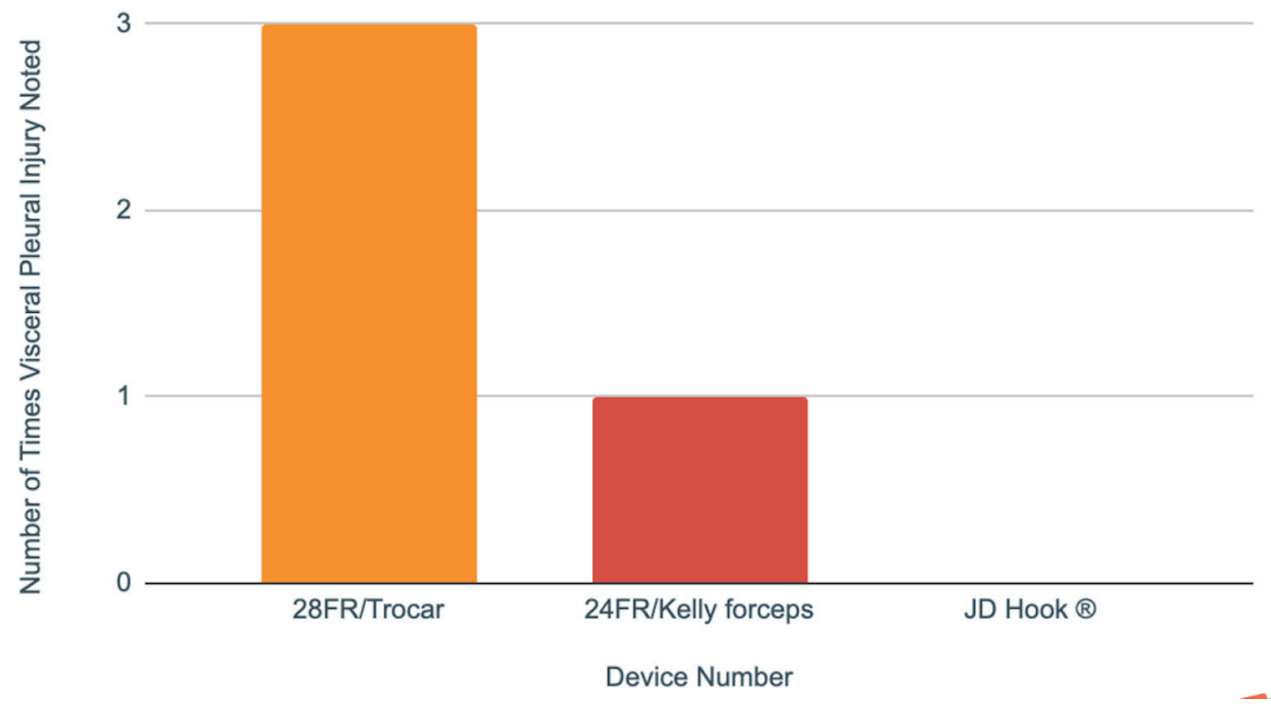

Figure 3. Lung injury score shows number of times that the pleural surface contact was noted during chest tube insertion (out of three consecutive trials for each device).

narios, (where timely access to chest cavity can mean the difference between life and death), by minimizing insertion steps and procedure complexity so that it can be safely performed by "field-skilled" providers including emergency medical responders, firefighters, and combat zone nursing staff. For that purpose, we have defined a "triangle of safety" zone (Figs. 5, 6 ), which in our opinion, represents the safest chest tube place-
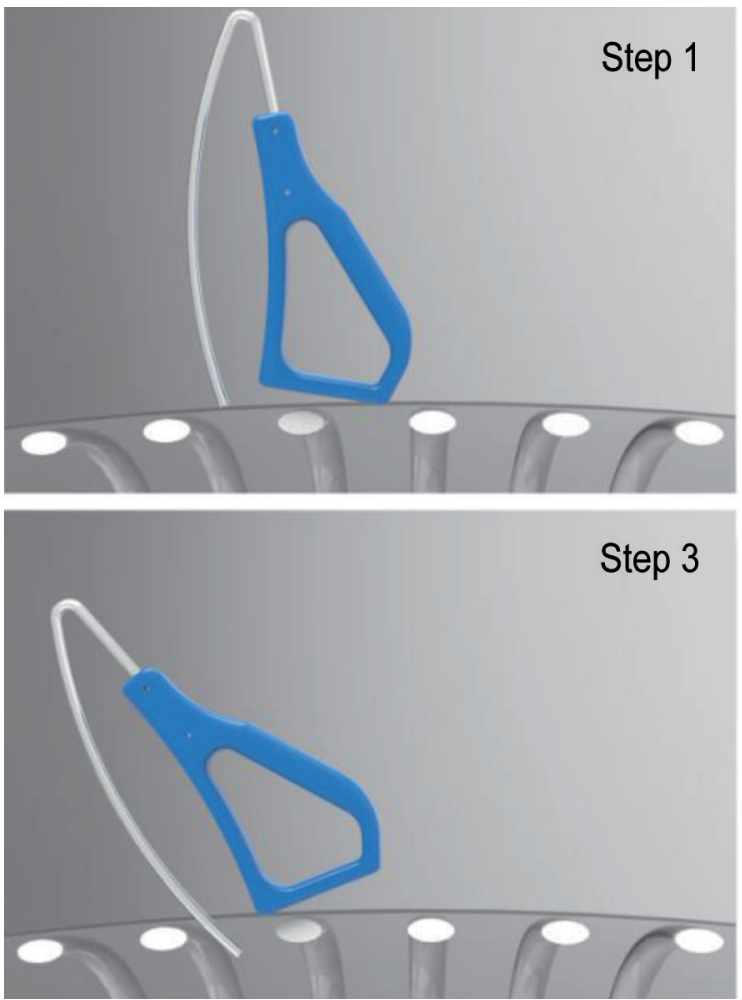

ment zone in various environments. This zone, hereby identified as "JD safety triangle" is located on each anterior hemi thorax, extending from the second intercostal space in the midclavicular line, extending laterally to the sixth intercostal space at the midaxillary line, from where it extends medially from mid axillary to anterior axillary line (within sixth intercostal space), and then extending cephalad to the second intercostal
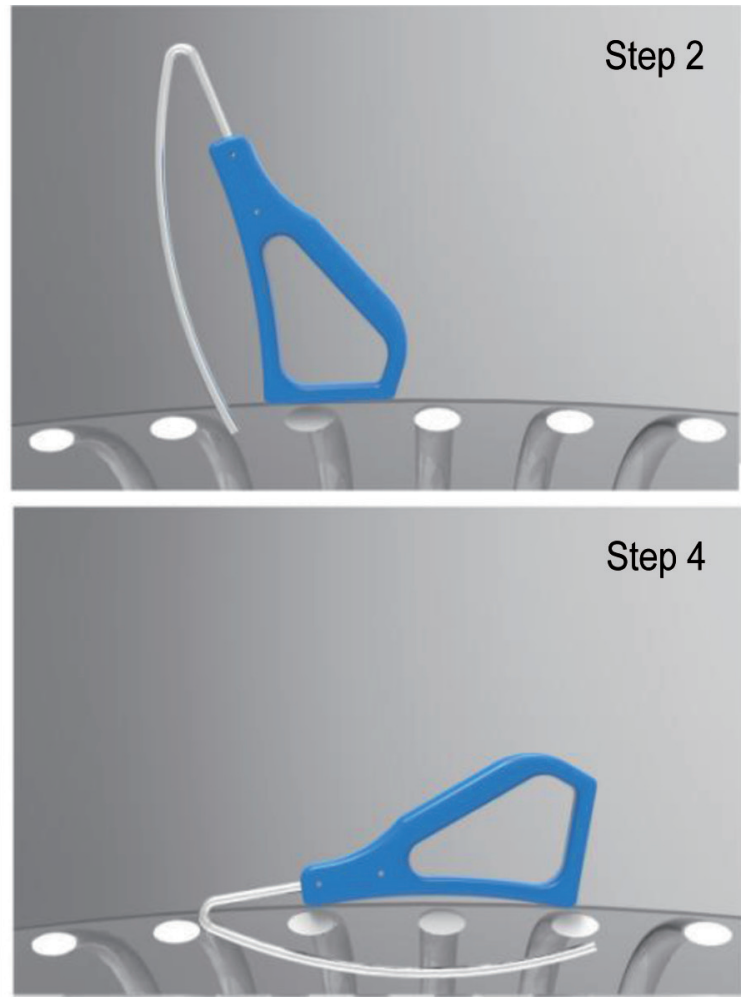

Figure 4. Schematic drawing showing simplified insertion steps for the JD Hook ${ }^{\circledR}$ chest tube insertion device. Tip angulation and base specifics minimize risk of lung injury during the insertion process. 


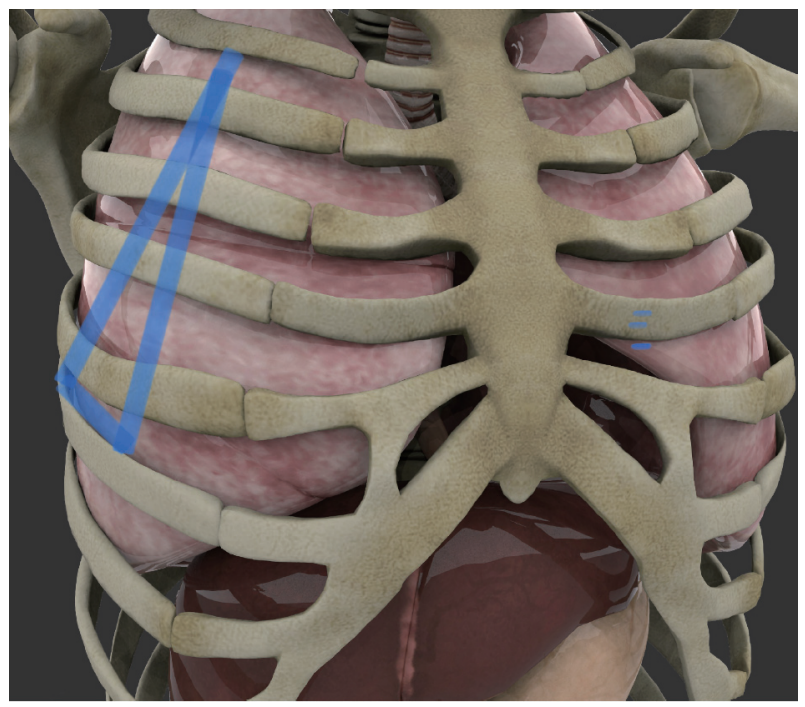

Figure 5. Outline of JD Hook ${ }^{\circledR}$ placement safety zone on right ribcage between the second and sixth intercostal space (original artwork depicting the JD safety zone, by J. Durrani, overlaid on Shutterstock Image ID: 1321108376, used with permission and standard use license).

space within the midclavicular line.

Our data show that in vitro model, JD Hook ${ }^{\circledR}$ device is a safer and faster chest tube insertion device compared to the currently available chest tube devices. The data confirm that the new device can be inserted within a significantly shorter period of time compared to conventional devices. Due to built in safety design characteristics, and using the JD safety triangle points for guidance, it is anticipated that there would be lesser dependence on operator skill level for safe and timely placement of chest tubes in the field compared to the conventional devices. Further studies with animal models and operators with more variations in skill sets, will help to confirm the clinical usefulness of this new device.

\section{Conclusions}

JD Hook ${ }^{\circledR}$ device is a safer and faster chest tube placement device compared to conventional devices when used within the JD safety triangle. Due to its ease of use and safety features, the device can be used in unconventional settings like field trauma stations and combat zones. Further studies with animal models and operators with more variations in skill sets, would be needed to confirm the clinical usefulness of this new device.

\section{Acknowledgments}

We gratefully acknowledge professional assistance by Matthew Sommerfield and Michael Roumeliotis, of MTS Ventures (https://www.mts-designmfg.com), for manufacturing the prototype device. We also acknowledge Jason Konzelmann, director of Desales SIM Labs (https://www.desales.edu/academ-

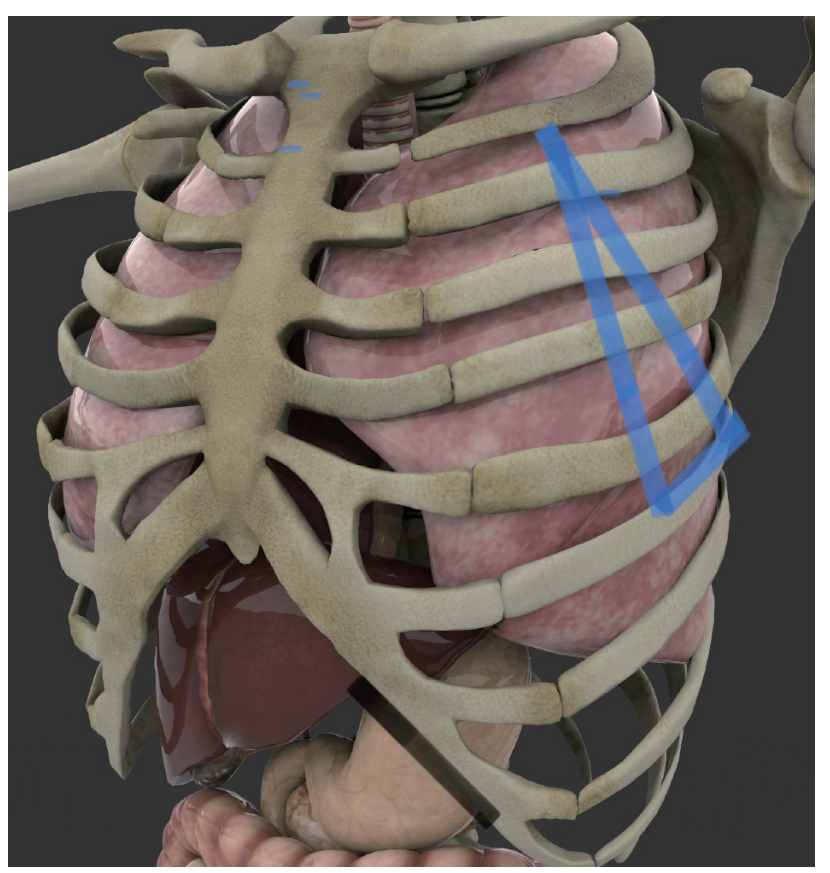

Figure 6. Outline of JD Hook ${ }^{\circledR}$ placement safety zone on left side. Base of the safety triangle rests at the sixth intercostal space between the midaxillary and anterior axillary line (original artwork depicting the JD safety Zone, by J Durrani, overlaid on Shutterstock Image ID: 1321108361, used with permission and standard use license).

ics/academic-divisions-departments/division-of-healthcare/ healthcare-simulation-lab) for providing facility support to complete the study.

\section{Financial Disclosure}

Study was funded solely by JFD-PK Medical LLC. Jameel Durrani is the owner of JFD-PK Medical LLC, and patent holder of the JD Hook ${ }^{\circledR}$ Pleural Access device. No additional public or institutional funding was utilized for this study.

\section{Conflict of Interest}

Figures 5 and 6 contain original artwork by the author overlaid on Shutterstock Images used under the terms of standard license.

\section{Informed Consent}

Not applicable.

\section{Data Availability}

Complete data supporting the findings of this study (including video monitoring of the procedure insertion times data) 
are available from the corresponding author upon reasonable request.

\section{References}

1. Kuckelman J, Cuadrado D, Martin M. Thoracic Trauma: A Combat and Military perspective. CurrTrauma Rep. 2018;4:77-87.

2. Mancini MC. Blunt chest trauma. Emedicine. http:// emedicine.medscape.com/article/428723-overview.

3. Defense USDoD. Tactical combat casualty care (T-CCC) guidelines for medical personnel. 2016;v03.

4. Martin M, Satterly S, Inaba K, Blair K. Does needle thoracostomy provide adequate and effective decompression of tension pneumothorax? J Trauma Acute Care Surg. 2012;73(6):1412-1417.

5. Fortune JB, Murphy S, Tiller K. Optimal initial positioning of chest tubes to prevent retained hemothorax using a novel steerable chest tube with extendable infusion cannula. Mil Med. 2021;186(Suppl 1):324-330.

6. United State patent and Trademark Office (USPTO): Application \# 15/350,880. Patent \# US 10,137,284, B2. Date Issued: Nov 27, 2018.

7. MTS Ventures (Prototype design and manufacturing), 905 Harrison Street, Suite 136, Allentown, PA 18103.

8. DeSales University Healthcare Simulation Lab, Gambet Center, 2755 Station Ave, Center Valley, PA18034. 